\title{
Introducing the inverse hoop conjecture for black holes
}

\author{
Shahar Hod ${ }^{1,2, a}$ \\ ${ }^{1}$ The Ruppin Academic Center, 40250 Emeq Hefer, Israel \\ 2 The Hadassah Academic College, 91010 Jerusalem, Israel
}

Received: 23 November 2020 / Accepted: 2 December 2020 / Published online: 13 December 2020

(C) The Author(s) 2020

\begin{abstract}
It is conjectured that stationary black holes are characterized by the inverse hoop relation $\mathcal{A} \leq \mathcal{C}^{2} / \pi$, where $\mathcal{A}$ and $\mathcal{C}$ are respectively the black-hole surface area and the circumference length of the smallest ring that can engulf the black-hole horizon in every direction. We explicitly prove that generic Kerr-Newman-(anti)-de Sitter black holes conform to this conjectured area-circumference relation.
\end{abstract}

\section{Introduction}

The isoperimetric inequality [1] in a two-dimensional Euclidean space states that the area $\mathcal{A}$ of a connected domain is bounded from above by the simple relation

$\mathcal{A} \leq \mathcal{C}^{2} / 4 \pi$

where $\mathcal{C}$ is the circumference length of the two-dimensional domain. The equality in (1) may be attained by an engulfing circular ring.

On the other hand, the area $\mathcal{A}$ of a deformed (or wrinkled) two-dimensional patch which is embedded in a threedimensional space can violate the area-circumference relation (1) [2]. Likewise, the surface area of a $(3+1)$ dimensional black hole may in principle grow unboundedly with respect to its (squared) circumference length.

Intriguingly, however, it is well known that black holes in three spatial dimensions behave in many respects as twodimensional objects. In particular, a black hole is characterized by a thermodynamic entropy $[3,4]$ which is proportional to its two-dimensional surface area (and not to its effective volume). One can therefore expect that, in analogy with the two-dimensional relation (1), the surface area of a black hole may be bounded from above by a quadratic function of its circumference length.

The main goal of the present compact paper is to raise the inverse hoop conjecture, according to which the surface

a e-mail: shaharhod@gmail.com (corresponding author) areas of all stationary $(3+1)$-dimensional black holes are bounded from above by the simple functional relation

$\mathcal{A} \leq \mathcal{C}_{\mathrm{s}}^{2} / \pi$

where $\mathcal{C}_{\mathrm{s}}$ is the circumference length of the smallest ring that can engulf the black-hole horizon in all azimuthal directions [5-9].

\section{The inverse hoop conjecture in charged and spinning Kerr-Newman-(anti)-de Sitter black-hole spacetimes}

A Kerr-Newman-(anti)-de Sitter black-hole spacetime of mass $M$, angular momentum $J \equiv M a$, electric charge $Q$, and cosmological constant $\Lambda$ is characterized by the curved line element [10-13]

$$
\begin{aligned}
d s^{2}= & -\frac{\Delta_{r}}{\rho^{2}}\left(\frac{d t}{I}-a \sin ^{2} \theta \frac{d \phi}{I}\right)^{2} \\
& +\frac{\Delta_{\theta} \sin ^{2} \theta}{\rho^{2}}\left[\frac{a d t}{I}-\left(r^{2}+a^{2}\right) \frac{d \phi}{I}\right]^{2} \\
& +\rho^{2}\left(\frac{d r^{2}}{\Delta_{r}}+\frac{d \theta^{2}}{\Delta_{\theta}}\right),
\end{aligned}
$$

where the metric functions $\Delta_{r}, \Delta_{\theta}, \rho$, and $I$ are given by the functional expressions $[10,11]$

$$
\begin{aligned}
\Delta_{r} & \equiv r^{2}-2 M r+Q^{2}+a^{2}-\frac{1}{3} \Lambda r^{2}\left(r^{2}+a^{2}\right), \\
\Delta_{\theta} & \equiv 1+\frac{1}{3} \Lambda a^{2} \cos ^{2} \theta \\
\rho^{2} & \equiv r^{2}+a^{2} \cos ^{2} \theta
\end{aligned}
$$

and

$I \equiv 1+\frac{1}{3} \Lambda a^{2}$.

Asymptotically flat Kerr-Newman black holes are characterized by the simple relation $\Lambda=0$, whereas non- 
asymptotically flat Kerr-Newman-de Sitter and Kerr-Newman-anti-de Sitter black-hole spacetimes are characterized respectively by the relations $\Lambda>0$ and $\Lambda<0$. The horizon radii of the black-hole spacetime (3) are determined by the roots of the radial metric function $\Delta_{r}(r)[10,11,14]$. In particular,

$\Delta_{r}\left(r_{+}\right)=0$,

where $r_{+}$is the radius of the black-hole event horizon.

From Eqs. (3) and (8) one finds the compact expressions

$\mathcal{C}_{\mathrm{eq}}=2 \pi \frac{r_{+}^{2}+a^{2}}{r_{+} I}$

and

$\mathcal{A}=4 \pi \frac{r_{+}^{2}+a^{2}}{I}$

for the equatorial circumference and the horizon surface area of the Kerr-Newman-(anti)-de Sitter black hole.

Interestingly, from Eqs. (9) and (10) one finds the compact dimensionless ratio

$\mathcal{H}(M, Q, a, \Lambda) \equiv \frac{\pi \mathcal{A}}{\mathcal{C}_{\mathrm{eq}}^{2}}=\frac{I r_{+}^{2}}{r_{+}^{2}+a^{2}}$

for generic Kerr-Newman-(anti)-de Sitter black holes [15]. The conjectured inverse hoop relation asserts that stationary $(3+1)$-dimensional black holes are characterized by the simple relation

$\mathcal{H} \leq 1$

Taking cognizance of Eqs. (7) and (11), one finds that asymptotically flat Kerr-Newman black holes (with $\Lambda=0$ and therefore $I=1$ ) and Kerr-Newman-anti-de Sitter black holes (with $\Lambda<0$ and therefore $I<1$ ) conform to the inverse hoop relation (12). It is easy to show that KerrNewman-de Sitter black holes (with $\Lambda>0$ ) are characterized by the relation $\Lambda r_{+}^{2} \leq 1$ [16] and therefore also respect the inverse hoop relation (12).

\section{Summary and discussion}

The famous Thorne hoop conjecture [5] asserts that blackhole spacetimes of suitably defined mass $\mathcal{M}$ are characterized by the relation $\mathcal{M} \geq \mathcal{C} / 4 \pi$. Since there are many different definitions of mass (energy) in curved spacetimes, it is natural to ask: what is the exact physical meaning of the mass (energy) term $\mathcal{M}$ in the hoop relation? To the best of our knowledge, in his original work Thorne [5] has not provided a specific definition for the mass term $\mathcal{M}$ in the intriguing hoop conjecture.

In the present compact paper we have explicitly demonstrated that if the mass term $\mathcal{M}$ is interpreted as the irreducible mass $\mathcal{M}_{\text {irr }}$ of the black hole, then generic KerrNewman-(anti)-de Sitter black-hole spacetimes conform to the inverse hoop relation

$\mathcal{M}_{\text {irr }} \leq \mathcal{C}_{\mathrm{s}} / 4 \pi$

Taking cognizance of the fact that the irreducible mass of a black hole is related to its horizon surface area $\mathcal{A}$ by the simple relation

$\mathcal{M}_{\text {irr }} \equiv \sqrt{\mathcal{A} / 16 \pi}$

one realizes that the inverse hoop relation (13) is a statement about the geometric properties of the black-hole horizon, bounding its surface area in terms of the squared circumference of the smallest ring that can engulf the horizon in every direction:

$\mathcal{A} \leq \mathcal{C}_{\mathrm{s}}^{2} / \pi$

If true, the conjectured inverse hoop relation (15) implies that the black-hole surface area cannot be unboundedly wrinkled [17].

Finally, it is worth noting that there is an important numerical evidence [18] for the validity of the inverse hoop conjecture (15) in non-stationary (dynamical) black-hole spacetimes. In particular, in a very interesting work, East [18] has studied numerically the full non-linear gravitational collapse of self-gravitating spheroidal matter configurations. Remarkably, it has been explicitly demonstrated in [18] that, in accord with the weak cosmic censorship conjecture [19], the final state of the collapse is a black hole. Interestingly, the initially distorted dynamically formed horizons obtained in [18] are characterized by damped oscillations between being prolate and oblate (see Figure 1 of [18]).

Intriguingly, and most importantly for our analysis, the numerical data presented in [18] (see, in particular, Figure 1 of [18]) reveals the fact that, within the bounds of the numerical accuracy [20], the dynamically formed black holes presented in [18] are characterized by the relation

$\frac{\max \left\{\mathcal{C}_{\mathrm{eq}}(t), \mathcal{C}_{\mathrm{p}}(t)\right\}}{4 \pi \mathcal{M}_{\text {irr }}} \geq 1$,

where $\mathcal{C}_{\text {eq }}$ and $\mathcal{C}_{\mathrm{p}}$ are respectively the time-dependent (oscillating) equatorial and polar circumferences of the non-stationary black-hole horizons. Thus, the dynamically formed black holes presented in [18] seem to respect the conjectured inverse hoop relation (15). 
Acknowledgements This research is supported by the Carmel Science Foundation. I would like to thank Professor W. E. East for sharing with me his interesting numerical data. I would also like to thank Yael Oren, Arbel M. Ongo, Ayelet B. Lata, and Alona B. Tea for stimulating discussions.

Data Availability Statement This manuscript has no associated data or the data will not be deposited. [Authors' comment: I would like to emphasize that all relevant physical and mathematical calculations are explicitly presented in this paper.]

Open Access This article is licensed under a Creative Commons Attribution 4.0 International License, which permits use, sharing, adaptation, distribution and reproduction in any medium or format, as long as you give appropriate credit to the original author(s) and the source, provide a link to the Creative Commons licence, and indicate if changes were made. The images or other third party material in this article are included in the article's Creative Commons licence, unless indicated otherwise in a credit line to the material. If material is not included in the article's Creative Commons licence and your intended use is not permitted by statutory regulation or exceeds the permitted use, you will need to obtain permission directly from the copyright holder. To view a copy of this licence, visit http://creativecomm ons.org/licenses/by/4.0/.

Funded by SCOAP ${ }^{3}$.

\section{References}

1. See R. Osserman, Bull. Am. Math. Soc. 84, 6 (1978) and references therein

2. For a two-dimensional patch embedded in a three-dimensional space, the parameter $\mathcal{C}$ may be defined as the circumference length of the smallest ring that can engulf that patch in all directions

3. J.D. Bekenstein, Phys. Rev. D 7, 2333 (1973)

4. S.W. Hawking, Commun. Math. Phys. 43, 199 (1975)

5. K.S. Thorne, in Magic Without Magic: John Archibald Wheeler, ed. by J. Klauder (Freeman, San Francisco, 1972)

6. The mathematically compact and physically influential Thorne hoop conjecture [5] asserts that black-hole spacetimes are characterized by the relation $\mathcal{M} \geq \mathcal{C} / 4 \pi$. It is worth noting that the exact physical meaning of the mass (energy) term $\mathcal{M}$ in the hoop relation has not been specified in the pioneering work of Thorne [5]. In the present compact paper we shall explicitly demonstrate that if the mass term $\mathcal{M}$ is interpreted as the irreducible mass $\mathcal{M}_{\text {irr }} \equiv \sqrt{\mathcal{A}} / 16 \pi$ of the black hole, then generic Kerr-Newman(anti)-de Sitter black-hole spacetimes conform to the inverse hoop relation $\mathcal{M}_{\text {irr }} \leq \mathcal{C} / 4 \pi$
7. See $[8,9]$ and references therein for recent studies of the Thorne hoop conjecture [5]

8. S. Hod, Eur. Phys. J. C 78, 1013 (2018). arXiv:1903.09786

9. Y. Peng, Eur. Phys. J. C 79, 943 (2019)

10. B. Carter in Les Astres Occlus, ed. by B. DeWitt, C.M. DeWitt (Gordon and Breach, New York, 1973)

11. H. Suzuki, E. Takasugi, H. Umetsu, Prog. Theor. Phys. 100, 491 (1998)

12. We use natural units in which $G=c=\hbar=1$

13. Here we use the familiar Boyer-Lindquist spacetime coordinates $(t, r, \theta, \phi)[10,11]$

14. Note that generic Kerr-Newman and Kerr-Newman-anti-de Sitter black-hole spacetimes are characterized by two (Cauchy and event) horizons, whereas generic Kerr-Newman-de Sitter blackhole spacetimes are characterized by three (Cauchy, event, and cosmological) horizons

15. Note that spinless $(a=0)$ black holes saturate the inverse hoop conjecture (2) [see Eqs. (7) and (11)]

16. Note that an extremal Schwarzschild-de Sitter black hole whose event horizon coincides with the cosmological horizon is characterized by the simple dimensionless relation $\Lambda r_{+}^{2}=1$

17. It is worth emphasizing that the conjectured inverse hoop relation (15) is expected to be valid only for black holes. In particular, it is straightforward to imagine non-black hole objects that violate the area-circumference relation (15). For example, a moon-like object whose surface is covered with craters can violate the relation (15). Likewise, a non-black hole Coronavirus-like object, whose surface is covered with spikes, can violate the area-circumference relation (15)

18. W.E. East, Phys. Rev. Lett. 122, 231103 (2019)

19. R. Penrose, Riv. Nuovo Cim. 1, 252 (1969)

20. W.E. East, Private communication 